\title{
Food Sensitization in Medically Resistant Chronic Rhinosinusitis with or without Nasal Polyposis
}

\author{
Mohannad Al-Qudah \\ Division of Otolaryngology, Department of Special Surgery, Jordan University of Science and Technology, \\ Irbid, Jordan
}

\section{Key Words}

Nasal polyposis $\cdot$ Rhinosinusitis $\cdot$ Serology $\cdot$ Food

sensitization · Sinus surgery · Atopy

\begin{abstract}
Background: Nasal polyposis is a common nasal mass with unknown etiology. It has been assumed that allergy predisposes to polyp formation. Our objective was to compare the prevalence of food sensitization in medically resistant chronic rhinosinusitis patients with or without nasal polyposis. Methods: One hundred and fifty-five patients who fulfilled the inclusion and exclusion criteria were incorporated into this study. The results of their total serum IgE and food-specific IgE levels were examined. Results: The average age was 33 years ( \pm 13 ) with 96 males and 59 females. The percentage of patients in each group that had a positive result for at least one tested allergen was $84 \%$ (88 patients in the sinusitis without polyposis group and 42 patients in nasal polyposis group). Patients without nasal polyposis reacted to an average of 4.6 foodstuffs, whereas patients with nasal polyposis reacted to 4.1. Egg white, sheefish and cherry were the most common type of sensitized food. There were no significant differences in the prevalence, type, number of positive food allergens and class level between the two groups. Conclusions: Food sensitization is common in medically resistant
\end{abstract}

chronic rhinosinusitis. Since food sensitization prevalence, type and severity do not significantly differ between the two groups studied, food atopy is unlikely to be a major factor in nasal polyposis pathogenesis.

(c) 2016 S. Karger AG, Basel

\section{Introduction}

Chronic rhinosinusitis (CRS) is a common disease with a significant morbidity and health care cost. It was estimated that CRS affects $11 \%$ of the total population in Europe [1]. The condition is treated medically with antibiotics, steroids, antihistamines and decongestant medications. Patients who fail to respond to optimal medical treatment [medically resistant (MR) CRS] are offered endoscopic sinus surgery; such sinusitis patients are the most commonly managed and followed group at otolaryngology clinics.

MRCRS is usually classified into CRS without nasal polyp (CRSsNP) and CRS with nasal polyp (CRSwNP). The etiology of nasal polyposis remains unknown and controversial. Eosinophils represent the most frequent inflammatory cell type in nasal polyposis, which has led to allergy being suspected in the etiology. However, a significant number of nasal polyposis patients have a nega-

Correspondence to: Dr. Mohannad Al-Qudah 
Table 1. Patient demographic features and clinical characteristics

\begin{tabular}{lccc}
\hline & $\begin{array}{l}\text { CRSsNP } \\
(\mathrm{n}=105)\end{array}$ & $\begin{array}{l}\text { CRSwNP } \\
(\mathrm{n}=50)\end{array}$ & p value \\
\hline Age, years & $33 \pm 13$ & $33 \pm 13$ & 0.9 \\
Male/female, $\mathrm{n}$ & $66 / 39$ & $30 / 20$ & 0.7 \\
Total serum IgE level, kU/l & $102(0-1,000)$ & $110(0-964)$ & 0.8 \\
Positive food serology test & $88(84)$ & $42(84)$ & 0.98 \\
Sum of specific IgE class, kU/l & $6.6 \pm 9$ & $5.9 \pm 9$ & 0.6 \\
Correlation between total serum IgE level and & 0.34 & 0.38 & $<0.001$ \\
$\quad$ number of positive foodstuff allergies & & 0.51 & $<0.001$ \\
$\quad \begin{array}{l}\text { Correlation between total serum IgE level and } \\
\quad \text { sum of specific IgE class }\end{array}$ & 0.3 & & \\
\hline
\end{tabular}

Values are presented as the mean $\pm \mathrm{SD}$, the mean (range), $\mathrm{n}(\%)$, or as stated.

tive inhalant allergic profile [2]. King [3] suggested a more probable role of food allergy in nasal polyposis pathogenesis than typical IgE-mediated inhalant allergies.

Pang et al. [4] detected a strong correlation between certain food allergies and nasal polyposis, and found $81 \%$ of patients with CRSwNP had a positive intradermal food allergy test. Another study identified a significant difference in the prevalence of food allergy between CRSwNP patients and healthy controls, with the food allergy test being positive in $72 \%$ of patients with CRSwNP [5]. Lill et al. [6] did not find such a high prevalence of food allergy among CRSwNP and suggested a positive role of milk allergy as a relevant pathogenetic entity in nasal polyposis. Based on these significant differences in the prevalence of food sensitization between healthy volunteers and CRSwNP patients, these studies [4, 5, 7] assumed a possible role of food allergy in nasal polyp etiology; however, a third group representing CRSsNP should be evaluated before such an assumption can be confirmed. The aim of this study was to compare the prevalence of food sensitization in MRCRS patients with or without nasal polyposis.

\section{Materials and Methods}

After obtaining approval from the institutional board committee at the King Abdullah University Hospital, Irbid, Jordan, the charts and medical records of consecutive CRS patients who failed to respond to optimal medical treatment and were offered endoscopic sinus surgery under the care of the author between July 2011 and December 2013 were reviewed. All patients had a detailed history, with clinical examination including nasal endoscopy and sinonasal CT scan. The exclusion criteria were a history of allergic fungus sinusitis, known cases of food allergy, granulomatous diseases, cystic fibrosis, primary ciliary dysfunction and sinonasal tumor.

Food Sensitization in CRS
Charts and electronic records were reviewed for demographic data, the presence and absence of nasal polyposis, total IgE levels, and serum concentrations of allergic-specific IgE to 34 food types measured by the Enzyme Allergo Sorbent Test, which was performed according to the manufacture's protocol (Euroimmune ${ }^{\circledR}$ kits; Medizinische Labordiagnostika, Lübeck, Germany). The results were graded into 6 classes: $0(<0.35$ kilounits of antibody per liter, $\mathrm{kU} / \mathrm{l}), 1$ (0.35-0.69 kU/l), 2 (0.7-3.5 kU/l), 3 (3.5-17.5 kU/l), $4(17.5-50 \mathrm{kU} / \mathrm{l}), 5(50-100 \mathrm{kU} / \mathrm{l})$ and $6(>100 \mathrm{kU} / \mathrm{l})$. Class 1 or more was defined as positive.

Parametric data were analyzed using the unpaired t test. Nonparametric data were analyzed using the Mann-Whitney $U$ test. Categorical data were analyzed by the $\chi^{2}$ test or Fisher's exact test, as appropriate. A nonparametric Spearman correlation was used to examine the relation between the total serum IgE level and number of positive foodstuff allergies, as well as the correlation between the total serum IgE level and sum of specific IgE class. A p value $<0.05$ was considered significant.

\section{Results}

A total of 155 patients were identified. The average subject age was 33 years $( \pm 13)$ and there were 96 males and 59 females. There were no significant differences between CRSwNP and CRSsNP patients based on age, gender distribution and total serum IgE level (table 1).

In each group, $84 \%$ of patients had a positive result for at least one of the tested allergens (88 patients in the CRSsNP group and 42 patients with CRSwNP). The average sum of class level in the CRSsNP subjects was 6.6, which compared to 5.9 in the CRSwNP group; this difference was also not significant. A positive significant correlation was found between total serum IgE level and the number of positive foodstuff sensitizations, as well as be- 


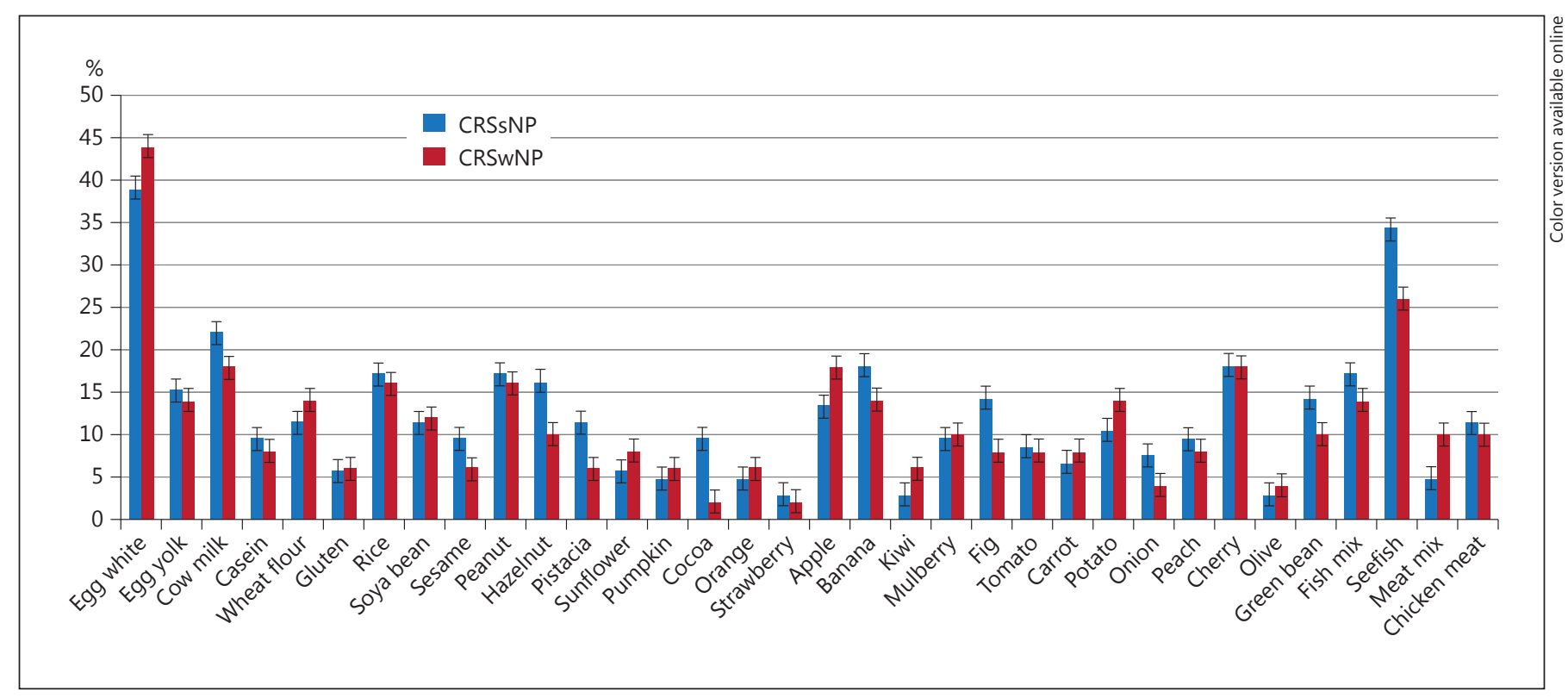

Fig. 1. The percentage of positive food allergies for each tested food in the two groups.

tween the total serum IgE level and sum of specific $\operatorname{IgE}$ class in both groups (table 1).

On average, each patient with CRSsNP had hypersensitization to 4.6 food types, whereas in patients with CRSwNP the number was 4.1; this difference was also not significant. Table 2 demonstrates the difference between the two groups based on the number of positive food sensitizations.

Egg white, sheefish and cherry were the three most frequent sensitized food types in both groups. Figure 1 shows the percentage of positive food sensitization for each tested food in both groups; there was no significant difference in this percentage between the 2 studies groups for each single tested food.

Nine CRSsNP patients (9\%) and 2 CRSwNP patients (4\%) had a serum concentration of specific IgE above class 3. Figure 2 shows the distribution of patients in both groups based on the highest class level. Again, the differences were not significant.

\section{Discussion}

Our results are consistent with previous findings of a high prevalence of food sensitization in chronic sinusitis patients $[4,5,7]$. Eighty-four percent of our MRCRS patients had at least one positive serology test for food sensitization; however, we could not find any significant differ-

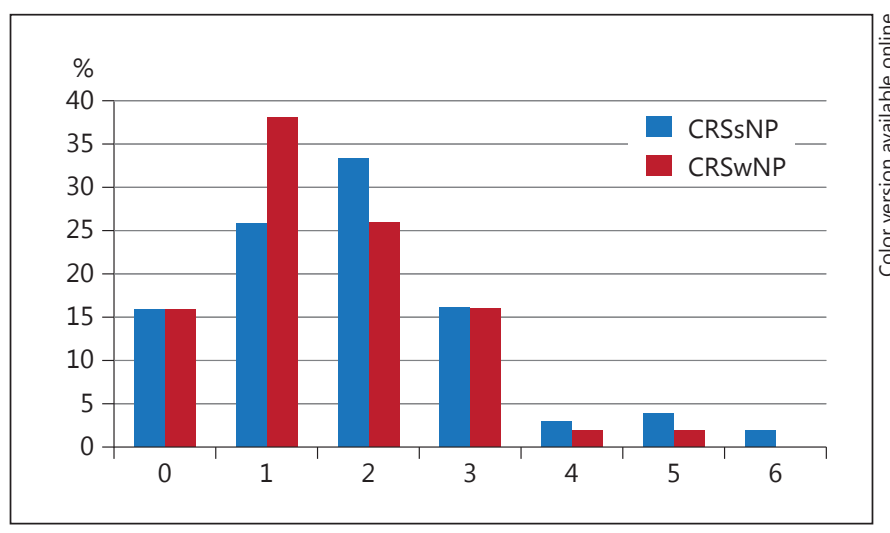

Fig. 2. The distribution of patients based on the highest class level in the two groups.

Table 2. Number of positive foodstuff allergens in each group

\begin{tabular}{lccc}
\hline $\begin{array}{l}\text { Number of } \\
\text { positive allergens }\end{array}$ & CRSsNP, \% & CRSwNP, \% & p value \\
\hline 0 & 15 & 16 & 0.92 \\
1 & 17 & 30 & 0.22 \\
2 & 20 & 14 & 0.35 \\
3 & 8 & 2 & 0.16 \\
4 & 5 & 8 & 0.43 \\
5 & 10 & 4 & 0.22 \\
6 or more & 21 & 18 & 0.47 \\
\hline
\end{tabular}

42

Int Arch Allergy Immunol 2016;169:40-44 DOI: $10.1159 / 000443737$ 
ences in the prevalence, type of food, number of food sensitizations and severity between CRSwNP and CRSsNP.

Pang et al. [4] studied the prevalence of food hypersensitivity in 80 nasal polyposis patients and 36 healthy volunteers using intradermal skin tests for 16 food allergens. They found a significant difference between the two groups: 65 with nasal polyps (81\%) compared to 4 controls (11\%) had a positive food reaction. They also found a significant difference in the median number of positive skin test results between the groups. The most common reactions were to wheat, potato and tomato. As only 1 patient reacted positively to food containing salicylate, they concluded that food allergy may play a more significant role in the pathogenesis of nasal polyposis than salicylate sensitivity.

In another prospective study, Doğru et al. [7] compared the prevalence of asymptomatic IgE-mediated food hypersensitivity in nasal polyposis patients with the prevalence of such food hypersensitivity in healthy controls using the epicutaneous prick test for 48 types of commonly consumed food. Of the 25 nasal polyp patients, $74 \%$ had a positive test result compared to $15 \%$ of the 20 cases in the control group. The mean number of positive results in the nasal polyp group was 10 , whereas the number in the control group was 4 . These two differences were statistically significant.

Collins et al. [5] found patients with nasal polyposis were no more likely to react to skin prick tests for inhalant allergens than controls, but were twice as likely to have positive intradermal food tests. Furthermore, only half of the patients with nasal polyposis and controls with positive food sensitization also had positive inhalation sensitization. Regardless of whether or not the patients with nasal polyposis had positive or negative inhalation hypersensitivity, they still had the same likelihood of having positive food sensitization.

In our study, the most commonly found sensitized food reactions (egg white, sheefish and cherry) differed from those reported by others $[4,5,7]$. This discrepancy may reflect different nutritional habits, genetic variations or studied food allergens. We did not find a significant difference in the type of positive food sensitization between MRCRS patients with or without nasal polyposis. Lill et al. [6] evaluated the relationship of wheat and milk allergies to nasal polyposis using radioallergosorbent tests. They found that 12 and $14 \%$ of nasal polyposis patients exhibited a wheat and milk allergy, respectively, compared to 14 and $0 \%$ in the control group. They concluded that cow milk allergy but not wheat allergy might be a relevant pathogenetic entity in nasal polyposis. We observed cow milk sensitization in $18 \%$ and wheat allergy in $14 \%$ of CRSwNP patients.
Studies have suggested that after the age of 2 years the prevalence of food allergies decreases. However, Bhombal et al. [8] found a higher prevalence of food allergy in children older than 2 years of age versus those younger than 2 years in consecutive pediatric patients undergoing otolaryngology procedures. They suggested this variation may reflect a higher prevalence of food allergy in the otolaryngology population.

Many studies have described a possible association between food allergy and certain medical disorders. About $35 \%$ of children with atopic dermatitis have a food allergy based on double-blind, placebo-controlled food challenges [9]. Removal of a specific food allergen may lead to a reduction or resolution of clinical symptoms in affected patients; reintroduction of the food may then exacerbate the atopic dermatitis if it is food responsive $[10,11]$. The rate of food-induced bronchospasm in asthmatic patients has been reported as $68 \%$ [12].

The prevalence of food allergy in the general population varies according to the method used (self-report, testing, physician evaluation), geographic region and foods included in the assessment [13]; however, it has been estimated to be $5-6 \%$ in infants and young children, and 3.7\% in adults [14]. This high prevalence of positive food sensitization in MRCRS patients is unlikely to reflect a general atopic diathesis of studied patients and it suggests a specific response to foodstuffs. A prospective study with a control group would be helpful to determine the comparative food sensitization prevalence in healthy subjects and chronic sinusitis patients with or without polyposis.

Recent reports have suggested a similar sensitivity and specificity in the serological measurement of food-specific IgE antibodies compared with skin-prick testing [15]. This test has become crucial for food allergy assessment [16]. Serological evaluation has the advantage of providing quantitative values that can help in predicting with high certainty the presence of a clinically significant food allergy [16]. This decreases the need for the gold standard test for food allergy diagnosis, the double-blind, placebocontrolled food challenge, which is time consuming and expensive. Additionally, avoidance is difficult with some foods, and compliance with exclusion diets is hard to ensure [17].

However, our results should be interpreted within the context of the diagnostic test performance. Sampson [18] concluded that skin tests and immunoassays for food allergy have an excellent negative predictive value, though less reliable positive predictive value. Indeed, further studies are required for greater exploration of the exact role of food allergy in sinusitis.

Int Arch Allergy Immunol 2016;169:40-44 DOI: $10.1159 / 000443737$ 
Elevation of total serum IgE levels is not specific to allergic diseases and can be caused by several medical disorders; however, many patients with allergic diseases have an elevation in their total IgE serum level. As there is no specific cutoff value that can determine patients with allergic disorders from those without, elevated total IgE cannot be used to make a diagnosis of allergy [19, 20]. Sunyer et al. [21] found that nonatopic patients with high levels of $\operatorname{IgE}$ are more likely to have asthma than those with normal IgE levels and reinforced previous evidence that asthma is associated with increased levels of total IgE, even in subjects negative for specific IgE to common aeroallergens, whereas in allergic rhinitis the measurement of total IgE has a low sensitivity and does not correlate with skin test reactivity or specific in vitro IgE testing, and even a normal IgE level does not rule out allergic rhinitis [22].

We found a weak correlation between total serum IgE and food-specific IgE levels. Mehl et al. [23] did not find determination of the ratio of food-specific IgE to total IgE to give better results (predicted probability, sensitivity and specificity) on which to base a food allergy diagnosis than the determination of food-specific IgE alone.

\section{Conclusion}

This retrospective review demonstrates a high prevalence of food sensitization in patients with MRCRS. However, no difference in the sensitized food profile between these patients based on the presence or absence of nasal polyposis was found. These findings suggest a possible role of food atopy in refractory chronic sinusitis, but not necessarily as a relevant pathogenesis in nasal polyposis development. Further studies may be warranted to explore the role of food sensitization in sinusitis.

\section{Acknowledgments}

I would like to thank those who assisted me in the collection of data, and the technical staff of the allergy/immunology lab at our hospital for performing laboratory tests.

\section{Disclosure Statement}

The author has no conflicts of interest to disclose.

\section{References}

1 Hastan D, Fokkens WJ, Bachert C, et al: Chronic rhinosinusitis in Europe - an underestimated disease: a GA ${ }^{2} L E N$ study. Allergy 2011;66:1216-1223.

2 Wilson KF, McMains KC, Orlandi RR: The association between allergy and chronic rhinosinusitis with and without nasal polyps: an evidence-based review with recommendations. Int Forum Allergy Rhinol 2014;4:93103.

3 King WP: Food hypersensitivity in otolaryngology: manifestations, diagnosis, and treatment. Otolaryngol Clin North Am 1992;25: 163-179.

4 Pang YT, Eskici O, Wilson JA: Nasal polyposis: role of subclinical delayed food hypersensitivity. Otolaryngol Head Neck Surg 2000; 122:298-301.

5 Collins MM, Loughran S, Davidson P, Wilson JA: Nasal polyposis: prevalence of positive food and inhalant skin tests. Otolaryngol Head Neck Surg 2006;135:680-683.

6 Lill C, Loader B, Seemann R, et al: Milk allergy is frequent in patients with chronic sinusitis and nasal polyposis. Am J Rhinol Allergy 2011;25:e221-e224.

7 Doğru H, Tüz M, Uygur K, Akkaya A, Yasan H: Asymptomatic IgE mediated food hypersensitivity in patients with nasal polyps. Asian Pac J Allergy Immunol 2003;21:79-82.
8 Bhombal S, Bothwell MR, Bauer SM: Prevalence of elevated total IgE and food allergies in a consecutive series of ENT pediatric patients. Otolaryngol Head Neck Surg 2006;134:578-580.

9 Eigenmann PA, Sicherer SH, Borkowski TA, Cohen BA, Sampson HA: Prevalence of IgEmediated food allergy among children with atopic dermatitis. Pediatrics 1998;101:E8.

10 Sampson HA, McCaskill CC: Food hypersensitivity and atopic dermatitis: evaluation of 113 patients. J Pediatr 1985;107:669-675.

11 Sampson HA, Scanlon SM: Natural history of food hypersensitivity in children with atopic dermatitis. J Pediatr 1989;115:23-27.

12 Bock SA, Atkins FM: Patterns of food hypersensitivity during sixteen years of doubleblind, placebo-controlled food challenges. J Pediatr 1990;117:561-567.

13 Sicherer SH: Epidemiology of food allergy. J Allergy Clin Immunol 2011;127:594-602.

14 Sicherer SH, Sampson HA: Food allergy. J Allergy Clin Immunol 2010;125:S116-S125.

15 Kurowski K, Boxer RW: Food allergies: detection and management. Am Fam Physician 2008;77:1678-1686.

16 Eckman J, Saini SS, Hamilton RG: Diagnostic evaluation of food-related allergic diseases. Allergy Asthma Clin Immunol 2009;5:2.

17 Asero R, Fernandez-Rivas M, Knulst AC, Knulst AC, Bruijnzeel-Koomen CA: Double- blind, placebo-controlled food challenge in adults in everyday clinical practice: a reappraisal of their limitations and real indications. Curr Opin Allergy Clin Immunol 2009;9:379-385.

18 Sampson HA: Food allergy. JAMA 1997;278: 1888-1894.

19 Stone KD, Prussin C, Metcalfe DD: IgE, mast cells, basophils, and eosinophils. J Allergy Clin Immunol 2010;125:S73-S80.

20 Klink M, Cline MG, Halonen M, Halonen M, Burrows B: Problems in defining normal limits for serum IgE. J Allergy Clin Immunol 1990;85:440-444.

21 Sunyer J, Antó JM, Castellsagué J, Soriano JB, Roca J: Total serum IgE is associated with asthma independently of specific IgE levels. The Spanish Group of the European Study of Asthma. Eur Respir J 1996;9:1880-1884.

22 Tschopp JM, Sistek D, Schindler C, et al: Current allergic asthma and rhinitis: diagnostic efficiency of three commonly used atopic markers (IgE, skin prick tests, and Phadiatop): results from 8,329 randomized adults from the SAPALDIA Study - Swiss Study on Air Pollution and Lung Diseases in Adults. Allergy 1998;53:608-613.

23 Mehl A, Verstege A, Staden U, et al: Utility of the ratio of food-specific IgE/total IgE in predicting symptomatic food allergy in children. Allergy 2005;60:1034-1039. 